\title{
Special Issue: Intelligent Management of Cloud, IoT and Big Data Applications
}

\author{
Claus Pahl • Muthu Ramachandran • Gary Wills
}

Received: 18 October 2019 / Accepted: 18 October 2019 / Published online: 14 November 2019

(C) Springer Nature B.V. 2019

Intelligent solutions are needed for today's complex, large-scale systems and applications. Big data is an example of this that refers to large datasets, characterized with high volume, variety, velocity, variability, veracity and complexity. Cloud and related distributed processing infrastructures such as fog and edge computing and the Internet of Things (IoT) produce and consume various kinds of application and system data at large scale, but also do so sometimes in resourceconstrained environments. Intelligent data processing can help with improved, automated self-adaptive systems management as well as better application processing.

This special issue connects intelligent analytics and processing technquies with their applications in cloud as well as supporting edge and IoT infrastructures. The issue focuses on intelligent solutions to large-scale computing problems, presenting new methods of analysis and processing for these application types. This emerging need to join intelligent processing in the presence of

C. Pahl $(\bowtie)$

Free University of Bozen-Bolzano, Bolzano, Italy

e-mail: cpahl@unibz.it

\section{Ramachandran}

Leeds Beckett University, Leeds, UK

e-mail: m.ramachandran@leedsbeckett.ac.uk

G. Wills

University of Southamption, Southamption, UK

e-mail: gbw@ecs.soton.ac.uk complexity and scale is addressed by this special issue covering a range of specific challenges in this context:

- Intelligent orchestration and scheduling of virtual computing and network resources in heterogenous, distributed and mobile cloud, edge and IoT architectures;

- Quality analysis and optimisation in cloud, edge and IoT systems including latency and resource costs;

- Machine learning and related intelligent analysis and processing techniques for cloud, fog, edge and IoT infrastructures;

- Algorithms, methods, quantitative measurement and experiments for open and distributed nextgeneration heterogeneous networks and architectures, using AI-based techniques for IoT and big data concerns;

This special issue has selected four papers after a vigorous paper review process following the journal guidelines and pleased to present the following papers:

1. Cloud-Based Multi-Agent Cooperation for IoT Devices Using Workflow-Nets by Moayad Aloqaily, et al. This paper addresses the problem faced by many complex scenarios where IoT devices have limited computational capabilities. Hence the need for these devices needing to outsource the computation to distributed but cooperating fog computing nodes. The paper presents a method for optimising the topology of the collaborating fog agents. The cooperating agents are represented as a Petri-net, the 
agents and their capabilities, and the workflows are represented mathematically. The mathematical representation enables the authors to propose a cooperation operator, with associated theorems and proofs. The practicality of the approach to provide time collaboration was then evaluated using simulation and then implemented with collaborating robots.

2. Optimizing Applications for Mobile Cloud Computing through MOCCA by Harun Baraki, et al. This paper presents a framework to aid developers decide on which components of the code can be offloaded from the mobile application, in order to boost performance while conserving resources. The framework takes into account the different hardware and networking capacities of various devices, so the it framework provides a flexible decision and offloading to different servers. The paper describes the algorithm for partitioning the code, the tool still requires the developer to make the required changes. The evaluations demonstrated the effectiveness of the framework and partition approach taken. The final decision demonstrates how this framework improves on previous work to reduce the development time in configuring the resource management, and improve the communication overhead with cloud.

3. Addressing Application Latency Requirements through Edge Scheduling by Atakan Aral, et al. This paper addresses the key issues of latency and availability of IoT based edge computing applications. To address these issues, the authors have developed a score-based edge service scheduling algorithm that evaluates network, compute, and reliability capabilities of edge nodes. In addition, they have also developed a set of simulation-based experiments on live video streaming services demonstrate significant improvements in both network delay and service time. You will find more inetesting applications from this paper.

4. Scheduling Algorithms for Heterogeneous Cloud Environment: Main Resource Load Balancing Algorithm and Time Balancing Algorithm by Weiwei Lin, et al. This paper presents an interesting approach to Cloud-IoT load imbalacing research issue. This paper proposes two IoT aware multi-resource task scheduling algorithms for heterogeneous cloud environment namely main resource load balancing and time balancing. The algorithms aim to obtain better result of load balance, Service-Level Agreement (SLA) and IoT task response time and meanwhile to reduce the energy consumption as much as possible. Their results shows save in response time and energy efficiency of their proposed algorithm. You will find some interesting applications for Cloud and IoT integration.

5. A Typology of Online Privacy Personalities - Exploring and Segmenting Users' Diverse Privacy Attitudes and Behaviors by Eva-Maria Schomakers. The focus of this paper is an analysis of privacy management in online environments. The result of this analysis is a categorization of different user types. Based on surveys, both attitudes and behaviors are considered, resulting in a novel approach to user typologies that takes into account the users' privacy calculus, i.e., weighing benefits and concerns about data sharing.

Publisher's Note Springer Nature remains neutral with regard to jurisdictional claims in published maps and institutional affiliations. 